\title{
$\operatorname{CONF}-9505219-3$
}

Note: This is a preprint of a paper being submitted for publication. Contents of this paper should not be quoted nor referred to without permission of the author(s).

\section{COMPARATIVE DIAGNOSTICS OF ArF- and KrF- LASER GENERATED CARBON PLUMES USED FOR AMORPHOUS DIAMOND-LIKE CARBON FILM DEPOSITION}

\author{
A. A. PURETZKY*, D. B. GEOHEGAN**, \\ G. E. JELLISON Jr.**, AND M. M. McGIBBON*** \\ *Institute of Spectroscopy, Troitsk, Russia \\ **Oak Ridge National Laboratory, Oak Ridge, TN \\ ***University of Glasgow, Glasgow, United Kingdom
}

May 1995

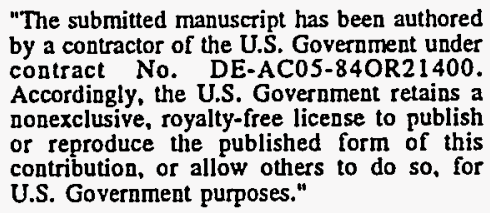

U.S. Government purposes."

Prepared by

Solid State Division

Oak Ridge National Laboratory

P. O. Box 2008

Oak Ridge, Tennessee 37831-6056

managed by

MARTIN MARIETTA ENERGY SYSTEMS, INC. for the

U.S. DEPARTMENT OF ENERGY

under contract DE-AC05-84OR21400

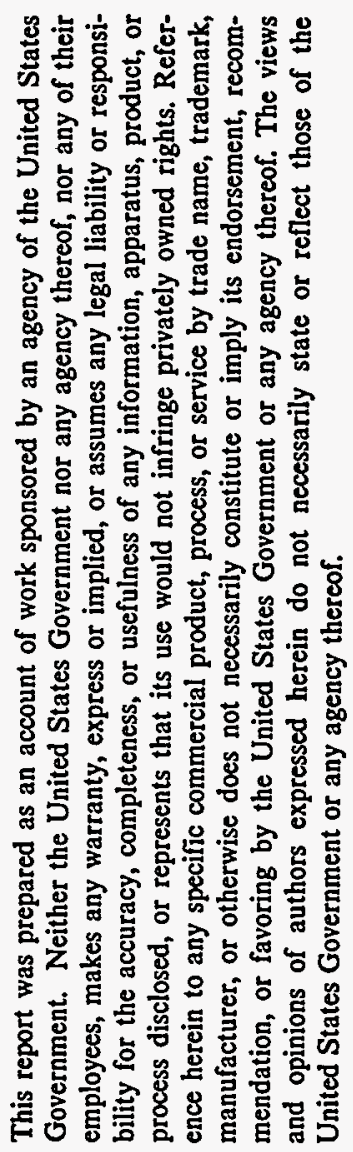




\author{
A.A. PURETZKY*, D.B. GEOHEGAN**, G.E. JELLISON Jr.**, AND \\ M.M. McGIBBON*** \\ *Institute of Spectroscopy, Troitsk, Russia \\ **Oak Ridge National Laboratory, Oak Ridge, TN \\ ***University of Glasgow, Glasgow, United Kingdom
}

\begin{abstract}
A comparative study of ArF- and $\mathrm{KrF}$-laser generated carbon plasmas has been performed under PLD conditions of amorphous diamond-like carbon (DLC) films. Gated-ICCD speciesresolved imaging, luminescence spectroscopy and ion probe diagnostics have revealed distinct differences between the carbon plumes generated by ArF- and KrF-lasers. KrF-laser (6.7 J/cm²) irradiation produces a less energetic carbon plasma containing larger amounts of luminescent $\mathrm{C}_{2}$ compared with ArF-laser ablation at the same energy fluence. Spectroscopic ellipsometry and EELS analysis of the DLC films deposited on $\mathrm{Si}<100>$ and $\mathrm{NaCl}$ substrates were utilized to characterize the high quality ArF- and $\mathrm{KrF}$-laser deposited films (up to $84 \%$ of $\mathrm{sp}^{3}$ bonded carbon for $7 \mathrm{~J} / \mathrm{cm}^{2}$-ArF-laser DLC film). The more energetic and highly-atomized ArF-laser carbon plasma appears to be responsible for the better diamond-like properties.
\end{abstract}

\title{
INTRODUCTION
}

Recently pulsed laser ablation of graphite with ultraviolet excimer laser wavelengths was found to permit the production of high quality amorphous diamond-like carbon (DLC) films. 1,2 Pulsed laser deposition (PLD) of carbon in high vacuum conditions with $\mathrm{KrF}-(248 \mathrm{~nm})$ and ArF-(193 nm) lasers permits the room-temperature production of amorphous diamond like films with smooth $(<20 \mathrm{~nm})$ surface morphology and high degree of diamond like character (large fraction of $\mathrm{sp}^{3}$ bonded carbon). The important practical feature of the UV-excimer PLD process is 
that much lower laser energy fluences $\left(5-20 \mathrm{~J} / \mathrm{cm}^{2}\right)$ are required compared to visible-and IR-laser PLD processing. ${ }^{2}$

In this work a comparative study of $\operatorname{ArF}-(193 \mathrm{~nm})$ and $\mathrm{KrF}(248 \mathrm{~nm})$ laser deposited diamond-like films has been performed and correlated with species resolved gated-ICCD imaging, optical spectroscopic and ion current probe diagnostics of the corresponding laser-generated carbon plasmas. The plasma diagnostics revealed several key differences between the plasmas generated by these two lasers. ArF-laser $\left(6.7 \mathrm{~J} / \mathrm{cm}^{2}\right)$ generated plasmas consist of a fast propagating ballshaped component of luminescence containing highly excited $\mathrm{C}^{++}, \mathrm{C}^{+}$, and $\mathrm{C}$ species followed by a slower component of cooler, atomized material $\left(\mathrm{C}, \mathrm{C}^{+}\right)$, followed by a third component which contains $\mathrm{C}_{2}$, and appears also to contain higher clusters and ultrafine particles. At the same fluence, the KrF-laser does not produce a luminescent plasma ball and appears to produce larger amount of luminescent $\mathrm{C}_{2}$, heavier clusters, and ultrafine particles at later times after the laser pulse. Higher quality DLC films were obtained using ArF-laser irradiation compared to KrF-laser PLD, as determined by EELS and spectroscopic ellipsometry analysis. The higher quality DLC films obtained with 193-nm ArF-laser irradiation appears correlated with both the smaller amount of clusters and ultrafine particles as well as with the higher kinetic energies of carbon ions and atoms generated by this laser.

\section{EXPERIMENTAL}

The experimental set up has been described previously.3,4 It consists of a stainless steel high vacuum chamber ( $40 \mathrm{~cm}$ diameter) equipped with Suprasil quartz windows for optical diagnostics. The chamber is pumped by a turbomolecular pump to a base pressure of $5 \times 10^{-7}$ Torr. A Questek (Model 2960) excimer laser operating on ArF (22 ns FWHM, 600 mJ) or KrF (28 ns FWHM, 900 $\mathrm{mJ}$ ) was used. The beam was apertured and focused into the chamber with a spherical lens (500 $\mathrm{mm}$ f.l. at $248 \mathrm{~nm}, 445 \mathrm{~mm}$ f.l. at $193 \mathrm{~nm})$ to a rectangular beam spot $(0.18 \mathrm{~cm} \mathrm{x} 0.11 \mathrm{~cm})$ at an incidence angle of $30^{\circ}$ onto $1^{\prime \prime}$-diameter pyrolytic graphite pellets (Specialty Minerals Inc., less than $10 \mathrm{ppm}$ total impurities). The pellets were rotated during the film deposition and plasma 
plume diagnostics experiments. The maximum laser fluences at the pellet surface were $7 \mathrm{~J} / \mathrm{cm}^{2}$ $(\operatorname{ArF})$ and $20 \mathrm{~J} / \mathrm{cm}^{2}(\mathrm{KrF})$.

Gated imaging was performed with an intensified charge-coupled device (ICCD), lenscoupled camera system (Princeton Instruments) with variable gain and gate width (5-ns minimum) and a spectral range from $200-820 \mathrm{~nm}$. A VariSpec TM tunable $(400-700 \mathrm{~nm})$ narrow band $(10 \mathrm{~nm}$ FWHM) liquid crystal filter (Cambridge Research \& Instrumentation, Inc.) was used for species resolved imaging.

Spectroscopic measurements of the plume luminescence were made with a 1.33-meter spectrometer (McPherson 209) equipped with an $1800 \mathrm{~g} / \mathrm{mm}$ holographic grating, an intensified, gated diode array (Princeton Instruments IRY-700RB) and a photomultiplier tube (Hamamatsu R955).

$\mathrm{N}$-type Si (100) wafers (resistivity $0.2-0.4 \mathrm{ohm}-\mathrm{cm}$ ) were used as substrates for PLD. The substrates were kept at room temperature and placed at variable distances from the target $(d=4-15$ $\mathrm{cm}$ ). $\mathrm{NaCl}$ crystals were also used as substrates for films deposited especially for EELS analysis.

Films were characterized by transmission electron microscopy (STEM), electron energy loss spectroscopy (EELS) and spectroscopic ellipsometry.

\section{RESULTS AND DISCUSSION}

\section{Carbon plume diagnostics}

To understand the differences between the ArF- and KrF-laser deposited DLC films, in situ diagnostics of the carbon plasma plume were performed under the respective film growth conditions. Figure 1 shows gated-ICCD images of the ArF- $\left(6.7 \mathrm{~J} / \mathrm{cm}^{2}\right.$ and $\mathrm{KrF}-(6.7$ and $\left.17.7 \mathrm{~J} / \mathrm{cm}^{2}\right)$ laser generated carbon plumes. Three main regions within the $A r F-\left(6.7 \mathrm{~J} / \mathrm{cm}^{2}\right)$ and $\mathrm{KrF}-\left(17.7 \mathrm{~J} / \mathrm{cm}^{2}\right)$ laser generated carbon plumes were discovered.

The first region noted [denoted 1 in Figs. 1 (a), (e)] has the highest emission intensity and characteristic ellipsoidal-ball shape. This plasma ball propagates with the average transport velocity, $v_{\mathrm{tr}}$, about $3.9 \mathrm{~cm} / \mu \mathrm{s}$ (ArF-laser, $6.7 \mathrm{~J} / \mathrm{cm}^{2}$ ) and $3.5 \mathrm{~cm} / \mu \mathrm{s}\left(\mathrm{KrF}-\right.$ laser, $17.7 \mathrm{~J} / \mathrm{cm}^{2}$ ) estimated from the propagation of the emission maximum. This region expands as well with a characteristic velocity, $\mathrm{v}_{\mathrm{exp}}=\mathrm{dR} / \mathrm{dt}=1.3 \mathrm{~cm} / \mu \mathrm{s}$ (estimated from 300 and $400 \mathrm{~ns}$ ArF-laser images). 
The species resolved ICCD imaging and luminescence were utilized to examine the species within this plasma ball. Figure 2 shows an example of species-resolved images taken at $\Delta t=500 \mathrm{~ns}$ with 100 ns gate. A few $10 \mathrm{~nm}$-width (FWHM) spectral regions were analyzed: $\mathrm{C}^{+}(427 \mathrm{~nm})$, $C(493 \mathrm{~nm}), C_{2}(516,470,560 \mathrm{~nm}), C_{3}(405 \mathrm{~nm})$ transitions, and a near-infrared region for monitoring the black-body emission from large clusters and ultrafine particles. The first plasma ball was observed in images with the tunable filter only at optical transition wavelengths corresponding to the $\mathrm{C}^{+}$resonance lines [Fig. 2(a)]. The luminescence study made with the spectrometer confirms this observation. Figure 3(a) shows the $\mathrm{C}^{+}, \mathrm{C}$ waveforms measured with the photomultiplier at $\mathrm{d}=2.15 \mathrm{~cm}$ from the target surface. The total emission time profile included in Fig. 3(a) was calculated from the set of ICCD imaging spatial intensity line-profiles at the same distance from the target surface. These emission waveforms show that $\mathrm{C}^{+}$and $\mathrm{C}$ species are located within the first plasma ball. At smaller distances from graphite target $(\mathrm{d}<1.0 \mathrm{~cm}) \mathrm{C}^{++}$ luminescence $\left(\lambda=229.7 \mathrm{~nm}\right.$, from $\left.145875 \mathrm{~cm}^{-1}\right)$ was also detected. The temporal peak of the $\mathrm{C}^{++}$ emission corresponded with that of $\mathrm{C}^{+}$at these distances, and the first component of neutral $\mathrm{C}$ emission. One can conclude from these data that the fast propagating ball [Figs. 1 (a)-(c), (e)-(g), and 2 (a)] is a propagating and recombining carbon plasma containing electronically excited species of $\mathrm{C}^{++}, \mathrm{C}^{+}$, and $\mathrm{C}$. Continual recombination results in the appearance of fast neutrals which appears as the first component of the C-luminescence waveform [Fig. 3 (a)]. Recombination also maintains the electron temperature within the plasma ball, slowing the drop of $T_{e}$ during the plasma expansion The average kinetic energy corresponding to the intensity maximum propagation of the first plasma ball can be estimated as $95 \mathrm{eV}$ (ArF-laser, $6.7 \mathrm{~J} / \mathrm{cm}^{2}$ ) and $77 \mathrm{eV}$ (KrF-laser, 17.7 $\left.\mathrm{J} / \mathrm{cm}^{2}\right)$.

The second region of the propagating plasma plume [2 in Figs. 1 (e)] appears in all of the images of Fig. 1., is characteristically round, and also propagates and expands. The maximum of emission intensity within this region propagates with the decreasing average velocity changing

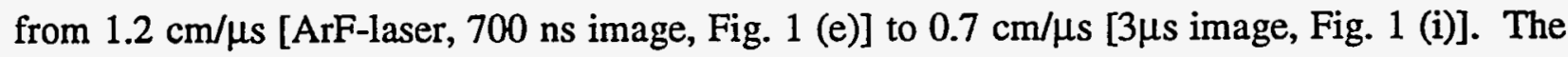
emission intensity in this region increases gradually to the red [Figs. 2 (c), (d)] and was observed at all wavelengths studied much weaker than that of the fast plasma ball (when present).. This continuous emision could be attributed to broadband emission from the large clusters and ultrafine 
particles present in the second plasma ball. A comparison of the ion current and $\mathrm{C}^{+}$emission time profiles in Fig. 3 (b) indicates that, in addition to the luminescent C-neutrals, non-emitting ground state ions are also present within the second ball of emission.

The third region of the plume appears near the target in all of the images at all wavelengths studied, but with remarkably higher intensity at the wavelengths corresponding to the $\mathrm{C}_{2}$ Swan bands [Fig. 2 (b)]. A characteristic "volcanic eruption" shape extending into the region 2 is observed above the upper surface of the region 3 [Fig. 2 (b)] when the carbon plume is imaged in the spectral windows corresponding to the $\mathrm{C}_{2}$ Swan bands.

A possible simplified picture for the formation of the observed three-region is as follows. The laser heating of the graphite target produces a hot region of evaporated carbon located near the target surface which contains $\mathrm{C}^{+}, \mathrm{C}$, clusters and ultrafine particles and droplets [Figs. 1 (j)-(n)]. This globule of hot carbon is moving relatively slowly $\left(0.4 \mathrm{~cm} / \mu \mathrm{s}, \mathrm{KrF}-1 \mathrm{aser}, 6.7 \mathrm{~J} / \mathrm{cm}^{2}\right)$ and ejects $\mathrm{C}, \mathrm{C}^{+}$, and carbon clusters forming the second plasma ball. The highly excited carbon clusters ejected from this hot globule can decompose and release the excited $C_{2}$ species which makes their "volcanic eruption" traces visible in $C_{2}$ luminescence bands. The ejected material propagates faster than the initial globule and forms the second plasma ball. An extensive clustering occurs within this ball during its propagation, expansion and cooling, which is consistent with the continuous emission observed from this region. The increase of the $\mathrm{KrF}$-laser fluence to approximately $17.7 \mathrm{~J} / \mathrm{cm}^{2}$ results in strong resonance enhanced multiphoton absorption of the laser radiation by neutral carbon vapor propagating on the leading front of the region 2 [Figs. 1 (j)-(n)] and formation of the hot plasma ball [Figs. 1 (a)-(d)]. The $193 \mathrm{~nm}$-ArF-laser ablation of graphite gives the picture very similar to that describe above except lower fluences are required to produce the first plasma ball and many fewer clusters are ejected from the hot near surface globule of carbon material [see Figs. 1 (e)-(i)].

\section{DLC films characterization}

The fraction of $\mathrm{sp}^{3}$-bonded carbon in the films was estimated using the EELS spectra. The spectra were obtained with a VG HB501 UX dedicated scanning transmission electron microscope 
(STEM) operated at an accelerating voltage of $100 \mathrm{KeV} .5$ The $30 \mathrm{~nm}$-thick DLC films for EELS analysis were deposited on $\mathrm{NaCl}$-substrates using $\mathrm{ArF}$-laser irradiation (11.1 Hz repetition rate, 4660 pulses, $5.6 \mathrm{~cm}$ substrate-target distance). The substrate was then dissolved in deionized water and the DLC-film was put on the STEM specimen copper grid. In order to estimate the fraction of $\mathrm{sp}^{3}$ bonded carbon, the EELS spectrum of crystalline carbon was measured as well.

The peak at $285.5 \mathrm{eV}$ corresponds to transitions from $1 \mathrm{~s}$ to $\pi^{*}$. The $1 \mathrm{~s}-\sigma^{*}$ transition is responsible for the higher energy peaks. In the amorphous DLC film the $1 s-\pi^{*}$ peak $(286.6 \mathrm{eV})$ is much smaller than that for the graphitized carbon sample due to the small amount of $\mathrm{sp}^{2}$-bonded carbon in the DLC film. The $1 s-\sigma^{*}$ peaks ( $>290 \mathrm{eV}$ ) in DLC films are broader compared to those in crystalline carbon because of the loss of structural order in the amorphous material. The $\mathrm{sp}^{3 /\left(\mathrm{sp}^{3}\right.}$ $+\mathrm{sp}^{2}$ ) ratio was estimated by the standard procedure 6 and found to be about $84 \%$ for the ArF-laser deposited film (on $\mathrm{NaCl}$-substrate). The low energy spectra also clearly demonstrated the amorphous diamond-like character of the deposited films, i. e., the $26 \mathrm{eV}$-plasmon peak was shifted to $30 \mathrm{eV}$ and the $6.7 \mathrm{eV}-\pi-\pi^{*}$ antibonding transition could not be resolved because of the very low fraction of $\mathrm{sp}^{2}$ bonded carbon.

The optical properties of both ArF- and KrF-laser deposited films were measured by spectroscopic ellipsometry. These measurements were performed on a two-channel polarization modulation ellipsometer ${ }^{7}$ which measures three of the associated ellipsometric parameters, $\mathrm{N}=\cos 2 \psi, \mathrm{S}=\sin 2 \psi \sin \Delta$ and $\mathrm{C}=\sin 2 \psi \cos \Delta(\psi$ and $\Delta$ are the ellipsometric angles) simultaneously in a single scan. The spectral range investigated was $220-840 \mathrm{~nm}$. The real and imaginary parts of the complex refractive index were obtained by fitting the experimental curves using a fiveparameter model developed by Forouhi and Bloomer for amorphous semiconductors ${ }^{8}$ (for details see Ref. 9). Two films, one deposited by $\mathrm{KrF}$-laser and one by ArF-laser, were analyzed. For the $\mathrm{KrF}$-laser film ( $380 \mathrm{~nm}$ maximum thickness measured by profilometry), the best fit $\left(\chi^{2}=0.7\right)$ to the experimental data was obtained with the following sequence of layers: (1) rough layer (0.74-nm), (2) DLC film (327.1 nm), (3) interface layer (14.5 nm), and finally c-Si. The same layer model applied to the ArF-laser generated film (127 nm maximum thickness measured by profilometry) also gave a good fit to experimental data $\left(\chi^{2}=2.6\right)$. The derived thicknesses of the layers are: (1) 
$0.98 \mathrm{~nm}$, (2) $110.4 \mathrm{~nm}$ and (3) $3.9 \mathrm{~nm}$, respectively (see above). The $\mathbf{n}$ values measured for this films changed from 2.56 to $2.74(\mathrm{KrF})$ and 2.52 to $2.77(\mathrm{ArF})$ in the spectral range studied. By comparison, the $n$ value for diamond varies from 2.40 to 2.66 over the same spectral region. The lower absorption coefficient for the ArF-laser DLC (versus the $\mathrm{KrF}$-laser film) corresponds with the trend noted during the entire series of runs, i.e., (a) higher electrical resistance and (b) more transparent diamond like films obtained with the ArF-laser compared to those deposited using the KrF-laser. It should be underlined that the higher-quality ArF-laser DLC was achieved with nearly 3-times less laser power compared to that used with the $\mathrm{KrF}$-laser, despite the general trend of higher quality DLC material with increasing laser energy for both wavelengths. The ellipsometry data also indicate substantial differences between the interfacial layer properties for these two cases. The refractive indices of ArF- and KrF-laser produced interfacial layers differ greatly $(\mathrm{n}=3$ and 2.4 at $2 \mathrm{eV}$, respectively). This indicates that the interfacial layers are quite different for these two cases.

\section{CONCLUSION}

A comparative study of $\mathrm{ArF}$ - and $\mathrm{KrF}$-laser deposited amorphous diamond like carbon (DLC) films has been performed in conjunction with relevant plasma diagnostics of the plasma plumes. ArF-laser irradiation produces the highest quality amorphous DLC films, with higher optical transmission, higher index of refraction, better electrical insulation, and higher $\mathrm{sp}^{3}: \mathrm{sp}^{2}$ ratios than $\mathrm{KrF}$-laser deposited DLC films. ArF-laser deposited films on $\mathrm{NaCl}$ substrates were estimated to contain $84 \% \mathrm{sp}^{3}$-bonded carbon. Unfiltered and species-resolved gated-ICCD imaging, spectroscopic, and ion probe current measurements show that the $\mathrm{KrF}$-laser generates substantially larger amounts of ultrafine particles and $C_{2}$ (and probably larger clusters) compared to that generated with the ArF-laser. In addition,the $\mathrm{KrF}$-laser generated plume at comparable fluences lacks the fast-propagating plasma ball noted using ArF irradiation. The $\mathrm{KrF}$ fluence must be

increased to at least $17 \mathrm{~J} / \mathrm{cm}^{2}$ to generate the similar fast component. However, spectroscopic ellipsometric analysis shows that the $\operatorname{ArF}\left(7 \mathrm{~J} / \mathrm{cm}^{2}\right)$ DLC films are still superior to the high-fluence $\mathrm{KrF}\left(17 \mathrm{~J} / \mathrm{cm}^{2}\right)$ DLC films. 


\section{ACKNOWLEDGMENTS}

The authors gratefully acknowledge Cambridge Research and Instrumentation, Inc. for the use of the tunable optical filter and also many helpful discussions with S.J. Pennycook and D. H. Lowndes. This work was supported by the Division of Material Sciences, U.S. Department of Energy under contract DE-AC05-84OR21400 with Martin Marietta Energy Systems, Inc., and NIS/ IPP program sponsored by Division of Defense Programs, U.S. Departement of Energy under contract DP-15. 


\section{REFERENCES}

1. D. L. Pappas, K. L. Saenger, J. Bruley, W. Krakov, J. J. Cuomo and R. W. Collins, J. Appl. Phys. 71, 5675 (1992).

2. F. Xiong, Y. Y. wang, V. Leppert, R. P. H. Chang, J. Mater. Res. 8, 2265 (1993).

3. D. B. Geohegan, Thin Solid Films 220,138 (1992).

4. D. B. Geohegan, Appl. Phys. Lett. 60, 2732 (1992).

5. N. D. Browning and S. J. Pennycook, Microbeam Analysis 2, 81 (1993).

6. S. D. Berger, D. R. McKenzie, P. J. Martin, Philosophical Magazine Letters 57, 285 (1988).

7. G. E. Jellison, Jr. and F. A. Modine, Appl. Optics 29, 959 (1990).

8. A. R. Forouhi and I. Bloomer, Phys. Rev. B 34, 7018 (1986).

9. G. E. Jellison, Jr., Thin Solid Films 234, 416 (1993).

10. D. F. Edwards and H. R. Philipp, Handbook of Optical Constants of Solids, ed. by E.D. Palic, Academic Press, San-Diego, 1985, p.665. 
Fig.1. Gated-ICCD photographs of the total visible carbon plasma emission generated by $\mathrm{KrF}-$ laser $\left(17.7 \mathrm{~J} / \mathrm{cm}^{2}\right)$ at (a) $\Delta \mathrm{t}=500 \mathrm{~ns}, 5 \mathrm{~ns}$-gate width, (b) ,700 ns, $5 \mathrm{~ns}$ (c), $1 \mu \mathrm{s}, 50 \mathrm{~ns}$, (d) $2 \mu \mathrm{s}$,

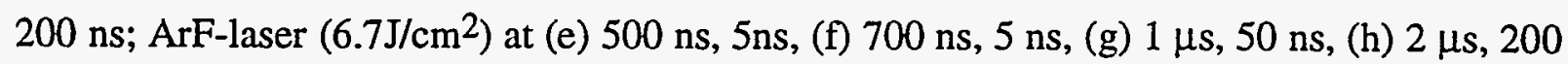
$\mathrm{ns}$, (i) $3 \mu \mathrm{s}, 200 \mathrm{~ns}$ and $\mathrm{KrF}-\operatorname{laser}\left(6.7 \mathrm{~J} / \mathrm{cm}^{2}\right)$ at (j) $500 \mathrm{~ns}, 5 \mathrm{~ns}$, (k) $700 \mathrm{~ns}, 50 \mathrm{~ns}$, (l) $1 \mu \mathrm{s}, 50 \mathrm{~ns}$, (m) $2 \mu \mathrm{s}, 200 \mathrm{~ns}$, (n) $3 \mu \mathrm{s}, 200 \mathrm{~ns}$. Three distinct spatial regions are indicated in (e) (see text). The 5-grayscale palette (shown at the bottom) is normalized to the number of counts which is indicated after the respective time delay for each image. Irradiation geometry is shown in the left upper corner.

Fig.2. Species resolved gated-ICCD images of the carbon plasma emission generated by KrF-laser $\left(17.7 \mathrm{~J} / \mathrm{cm}^{2}\right)$ at $\Delta \mathrm{t}=500 \mathrm{~ns}, 100 \mathrm{~ns}$-gate width. The corresponding wavelengths

are shown at the top.The 5-grayscale palette is normalized to the number of counts corresponding to the emission maxima of (a) the center of the first plasma ball (see text), (b)-(d) the third carbon plume region located near the target which is indicated at the bottom of each figure.

Fig.3. ArF-laser, $\left(6.7 \mathrm{~J} / \mathrm{cm}^{2}\right)$ generated (a) Luminescence of $\mathrm{C}^{+}(\lambda=426.7 \mathrm{~nm}$, originating from $168979 \mathrm{~cm}^{-1}$ above ground) and $\mathrm{C}\left(\lambda=247.9 \mathrm{~nm}, 61982 \mathrm{~cm}^{-1}\right.$ above ground) and the total emission calculated from the set of ICCD imaging spatial intensity line-profiles at $d=2.15 \mathrm{~cm}$ from the target surface. (b) Luminescence, total emission and ion current (peak current $0.3 \mathrm{~A}$ ) waveforms normalized to their maximum values measured at $4.0 \mathrm{~cm}$ from the target surface. The ion probe was biased to $-70 \mathrm{~V}$ (floating with respect to its shield). 


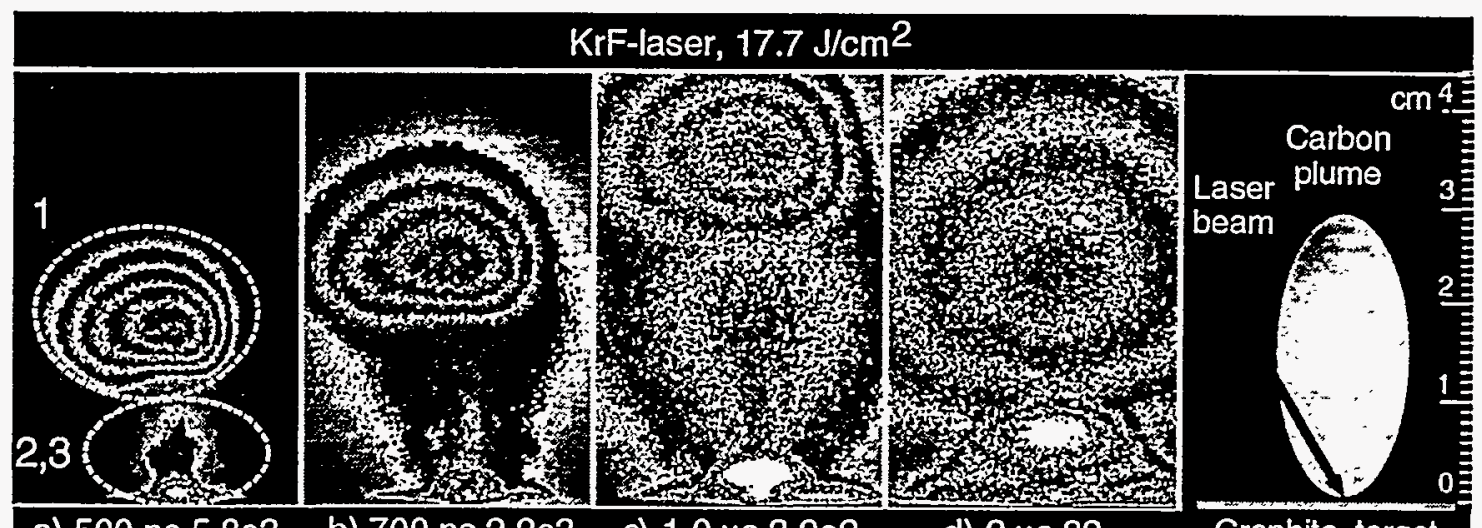

a) $500 \mathrm{~ns} 5.8 \mathrm{e} 3$

b) $700 \mathrm{~ns} 2.2 \mathrm{e} 3$

c) $1.0 \mu \mathrm{s} 3.9 \mathrm{e} 2$

d) $2 \mu s 82$

Graphite target

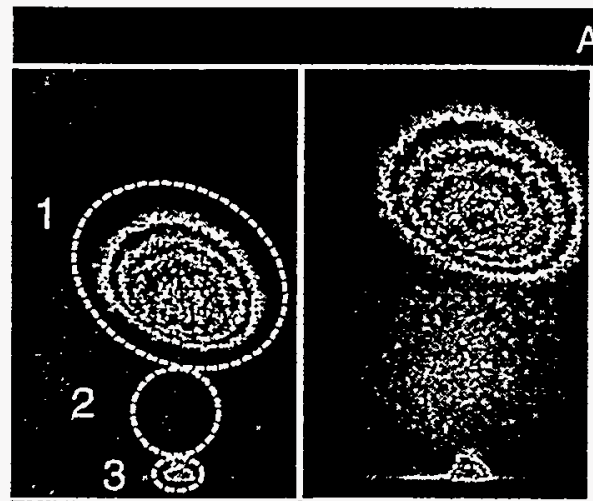

ArF-laser, $6.7 \mathrm{~J} / \mathrm{cm}^{2}$

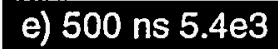

f) $700 \mathrm{~ns} 1.1 \mathrm{e}$
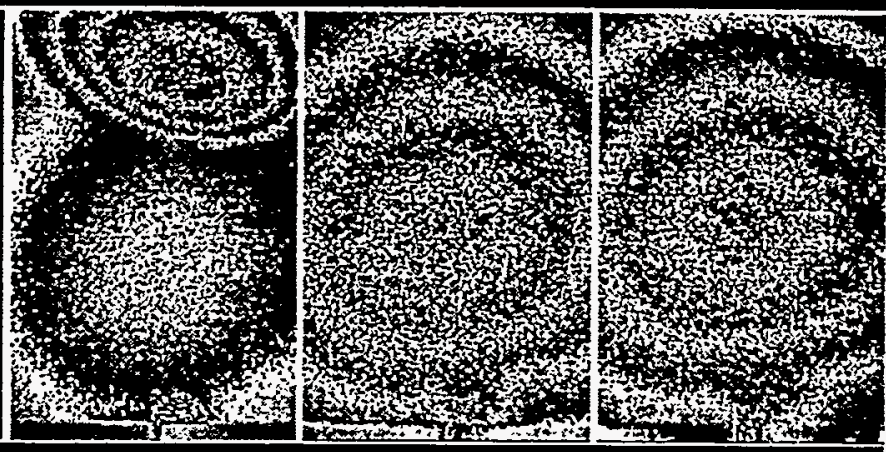

KrF-laser, $6.7 \mathrm{~J} / \mathrm{cm}^{2}$
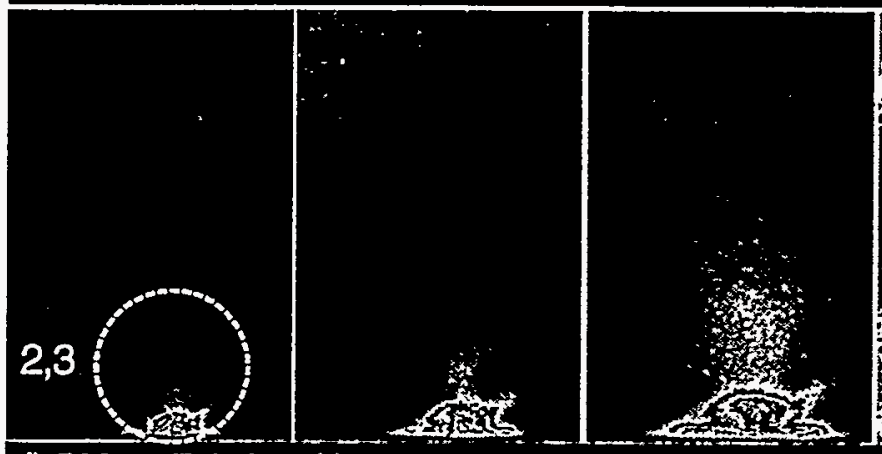

h) $2.0 \mu \mathrm{s} 50$

i) $3 \mu s 26$

j) 500 ns $7.4 \mathrm{e} 3$

k) $700 \mathrm{~ns} 1.9 \mathrm{e} 3$

l) $1.0 \mu \mathrm{s} 6.0 \mathrm{e} 2$

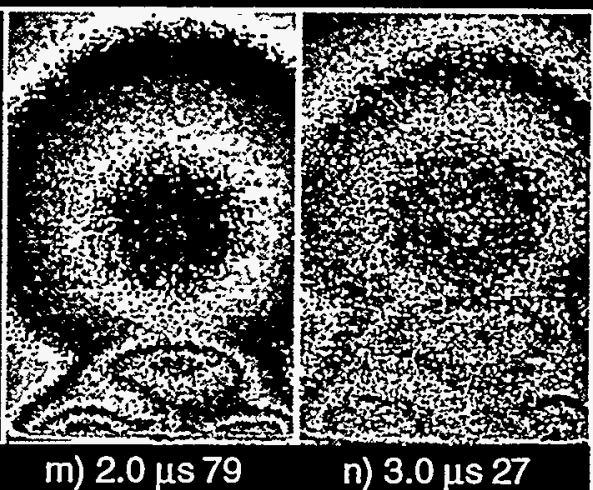

ret

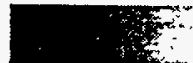

Palette

Fig. $1 /$ COLA $95 / 25 \%$ 


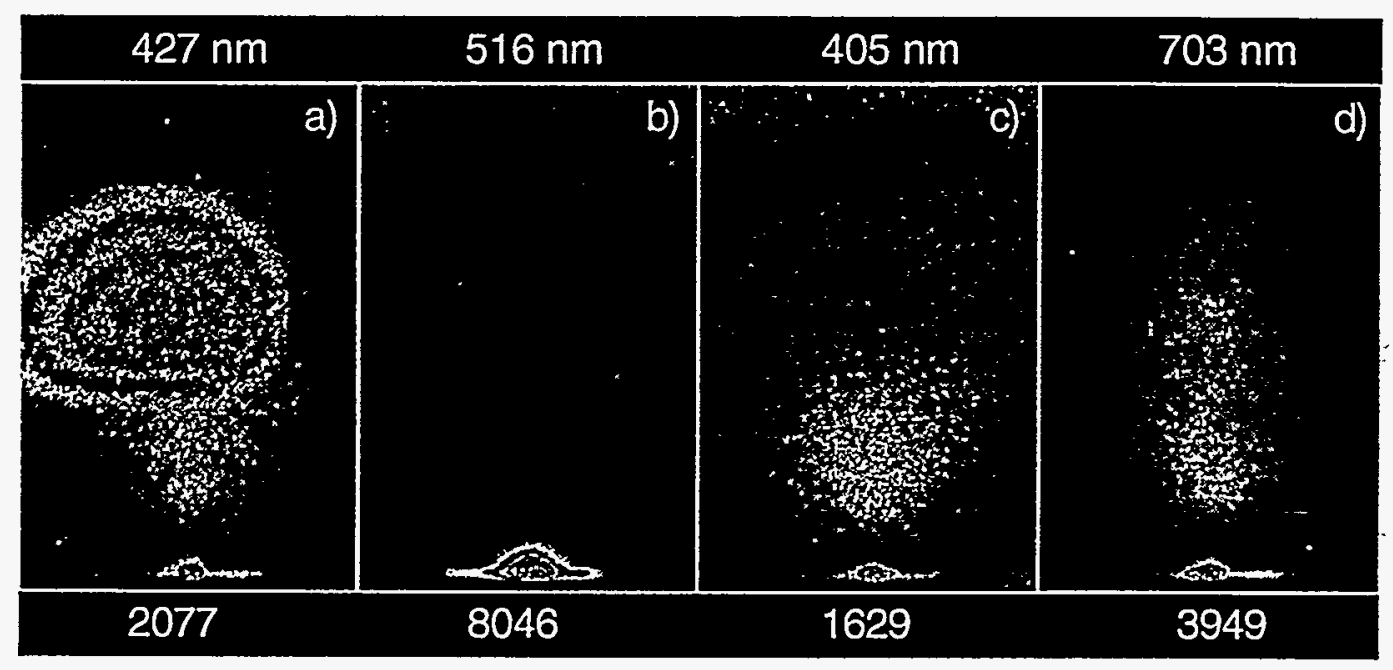

Fig. 2/COLA 95/25\% 


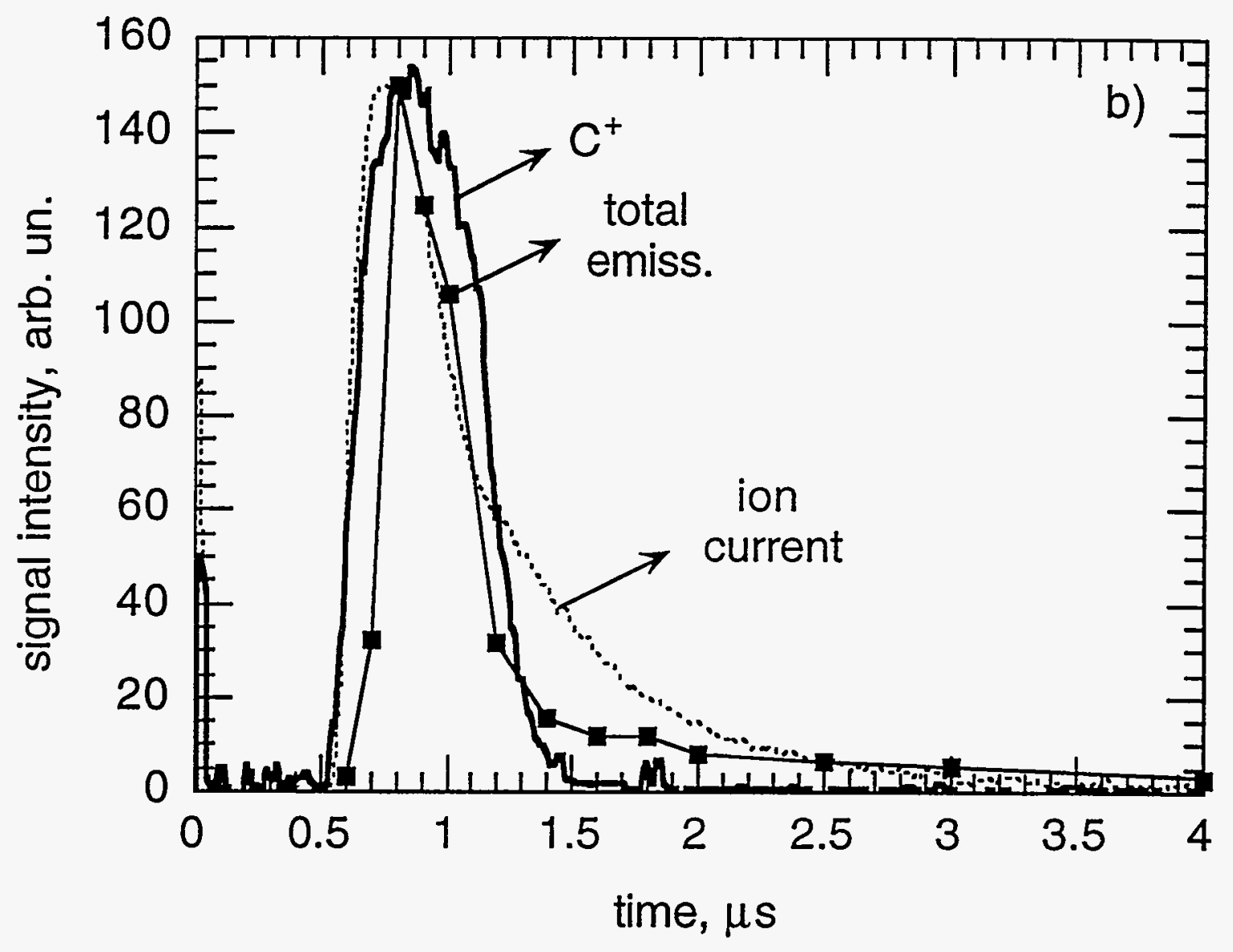

Fig. 3b/COLA 95/90\% 


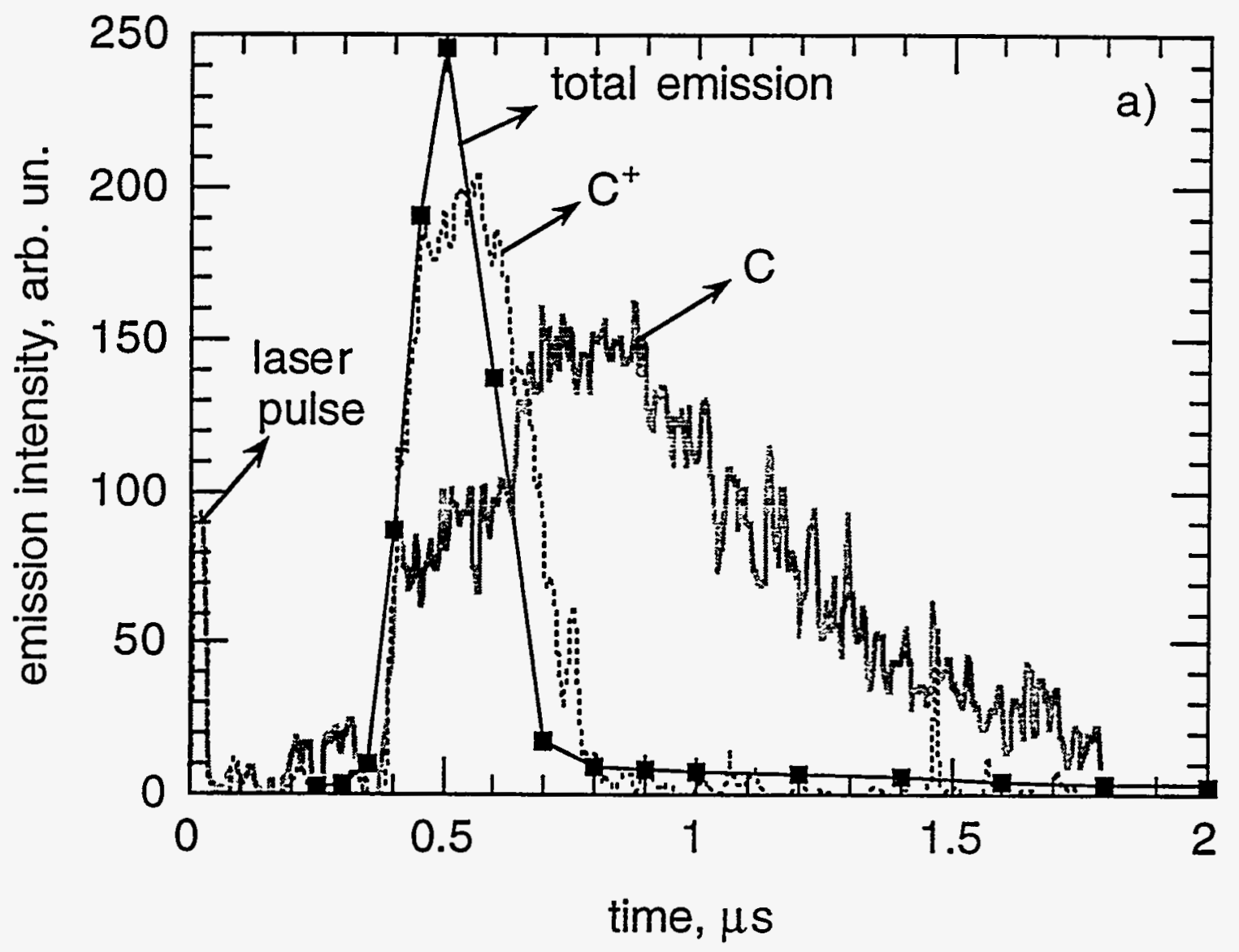

Fig. 3a/COLA 95/90\% 